\title{
Transcriptional activation of zebrafish cyp11a1 promoter is dependent on the nuclear receptor Ff1b
}

\author{
Sue Ing Quek and Woon Khiong Chan \\ Department of Biological Sciences, National University of Singapore, 14 Science Drive 4, Singapore, Republic of Singapore 117543 \\ (Correspondence should be addressed to W K Chan; Email: dbscwk@nus.edu.sg)
}

\begin{abstract}
The cytochrome P450scc (cholesterol side-chain cleavage enzyme) encoded by CYP11A1 catalyzes the first step in steroidogenesis by converting cholesterol to pregnenolone, and thus, controls the synthesis rate of steroid hormones. In mammals, steroidogenic factor 1 (SF1) has been implicated in the cAMP-mediated transcriptional activation of CYP11A1 promoter. In zebrafish, $\mathrm{Ff} 1 \mathrm{~b}$ has been established as the homolog of SF1. To assess the dependency of cyp11a1 expression on $\mathrm{Ff} 1 \mathrm{~b}$, the putative promoter of zebrafish cyp11a1, spanning $1.7 \mathrm{~kb}$, was isolated and bioinformatic analysis revealed two conserved FF1 response elements (FREs) that potentially bind Ff1b. Transfection studies in cell lines of different lineages confirmed that this promoter fragment contained the necessary regulatory elements required for its basal transcription. Truncation and mutagenesis studies performed in $\mathrm{Y} 1$ adrenocortical cells revealed that only the proximal FRE was essential for transcriptional activation. Electrophoretic mobility shift assay, however, indicated that Ff1b bound to both FREs, while their in vivo occupancy was confirmed using a chromatin immunoprecipitation assay. Lastly, the cyp11a1 promoter was able to direct EGFP expression specifically to the interrenal gland and genital ridge when transiently expressed in microinjected zebrafish embryos, and the promoter activity is potentiated by $f f 1 b$ overexpression as measured from luciferase reporter activity in zebrafish embryos.
\end{abstract}

Journal of Molecular Endocrinology (2009) 43, 121-130

\section{Introduction}

The biosynthesis of active steroid hormones from cholesterol in the adrenal cortex and the gonads involves five distinct steroid hydroxylases that belong to the cytochrome P450 gene family and two hydroxysteroid dehydrogenases (Payne \& Hales 2004). In teleost fish like zebrafish, cortisol, which acts as both glucocorticoid and mineralocorticoid, is synthesized in the adrenocortical homolog interrenal gland (Gallo \& Civinini 2003, Bury \& Sturm 2007). Although the active steroids in zebrafish are slightly different from those in mammals, the enzymes involved in steroidogenesis are identical. Among them, the cholesterol side-chain cleavage enzyme (P450scc, also known as CYP11A1) catalyzes the first and rate-limiting step in the steroidogenesis pathway by converting cholesterol to prenegnolone. Thus, the gene product of CYP11A1 occupies a key regulatory point in the biosynthesis of steroids (reviewed in Chung et al. (1997) and Guo et al. (2003, 2007)).

Steroid secretion and production are stimulated by adrenocorticotropin (ACTH) in the adrenals and by gonadotropins in the gonads. Acting through cAMP, they stimulate acute steroidogenesis by increasing the availability of cholesterol for CYP11A1 in the inner mitochondrial membrane (Jefcoate et al. 1987). cAMP also activates the transcription of steroid hydroxylases, leading to a sustained maintenance of steroidogenesis on a longer term basis (John et al. 1986). The transcriptional regulation of mammalian CYP11A1, including human (Morohashi et al. 1987), mouse (Rice et al. 1990), rat (Oonk et al. 1990), sheep (Pestell et al. 1993), and bovine (Ahlgren et al. 1999), has been intensively studied. Key cis-acting elements and their corresponding trans-acting factors, like steroidogenic factor 1 (SF1), AP-2, Sp1, TreP-132, and c-jun/AP-1, have been implicated in their transcriptional activation, particularly in relation to cAMP stimulation (Guo et al. 2007). The synthesis of steroid hormones is restricted to steroidogenic tissues and this is due to the developmentally important SF1, which is the central regulator responsible for tissue-specific expression of steroid hydroxylases, including CYP11A1 (Bakke et al. 2001, Kelly et al. 2004, Li et al. 2004). This also demands extensive coordination among the hypothalamus, pituitary, and steroidogenic tissues, and SF1 again acts to fully integrate the transcriptional responses needed for steroidogenesis (Hammer \& Ingraham 1999, Parker et al. 2002, Val et al. 2003, de Souza et al. 2006). Despite being well characterized in terms of gene and protein structure, expression profiles, post-translational 
modifications, interacting proteins, and its indispensable role in the regulation of steroidogenesis and the development of steroidogenic organs (Bakke et al. 2001, Val et al. 2003), very little is known about the molecular mechanism by which SF1 regulates its downstream target genes.

Fflb (Nr5ala), the ortholog of SF1 in zebrafish, is similarly the earliest known molecular marker for interrenal development (Chai \& Chan 2000). Ff1b also recognizes the same DNA response element as SF1 and LRH1 (Liu et al. 2003), both of which belong to the FF1 (NR5) subfamily of nuclear receptors. These DNA response elements will be termed as FF1 response elements (FREs) hereinafter. Knocking down fflb by antisense morpholinos completely disrupted the formation of interrenal gland, as characterized by the down-regulation of cyp11a1 transcripts and 3ß-HSD enzymatic activity (Chai et al. 2003, Hsu et al. 2003), and led to impaired osmoregulatory functions (Chai et al. 2003). These are reminiscent of findings from $\mathrm{SF}^{-/-}$ mice (Luo et al. 1994, Sadovsky et al. 1995). The similarity between the two organisms suggests a conserved regulatory program for adrenocortical development over 400 million years of evolution.

In view of the apparent conservation of the biosynthesis pathway for steroids between mammals and teleost (McGonnell \& Fowkes 2006, Bury \& Sturm 2007), it is reasonable to postulate that like mammalian $\mathrm{SF} 1$, Fflb is primarily responsible for regulating the steroid hydroxylases in zebrafish. Here, we report the cloning and characterization of the $5^{\prime}$ promoter region of zebrafish cyp11a1. The relative importance of the distal FRE (FREd) and proximal FRE (FREp) was addressed using deletion and site-directed mutagenesis analyses. The occupancy of the FREs by Ff1b was determined using electrophoretic mobility shift assay (EMSA) and chromatin immunoprecipitation assay (ChIP). Lastly, the ability of the cyp11a1 promoter to drive tissue-specific expression was examined in zebrafish embryos by transient transgenesis.

\section{Materials and methods}

\section{Cloning and sequence analysis}

The putative promoter region for the zebrafish cyp11a1 was isolated using a modified genome walking method (Siebert et al. 1995). Two microgram genomic DNA was separately digested with restriction enzymes that leave $3^{\prime}$ overhangs, namely, BstXI, NsiI, PstI, SacI, and SphI. After phenol/chloroform extraction, each pool of digested DNA genomic fragments was subjected to homopolymeric dC-tailing in a $40 \mu \mathrm{l}$ reaction containing $0.2 \mathrm{mM}$ dCTP, $10 \mathrm{mM}$ Tris-Cl ( $\mathrm{pH} 8 \cdot 4)$, $25 \mathrm{mM} \mathrm{KCl}, 1.25 \mathrm{mM} \mathrm{MgCl}_{2}$, and $38.4 \mathrm{U}$ terminal transferase for $1 \mathrm{~h}$ at $37^{\circ} \mathrm{C}$. The polymeric $(\mathrm{dC})$ tail served as a priming site for the anchored adapter primer, ANC1 (5'-GAGGTCGACGGGIIGGGIIGGGIIGH-3 $\left.{ }^{\prime}\right)$. Hemi-nested PCR was performed for each pool of genomic DNA fragments using the Expand Long Template PCR System (Roche) with ANC1 and gene-specific primers cyp1la1r2; 5'-CCATAATTCGG TGGACATTCCTG-3 ${ }^{\prime}$, followed by cyp11a1r3; 5'-TGAAGGCAAGGACACTGAGCAG-3' ${ }^{\prime}$. From $25 \mu \mathrm{l}$ primary amplified products, $1 \mu \mathrm{l}$ was used as template for secondary PCR. Amplified fragments were cloned using pGEM-T vector and sequenced. A $1.7 \mathrm{~kb}$ DNA fragment was recovered from the amplification of the SphI genomic DNA fragments. Potential transcription factor binding sites (TFBS) were determined using MatInspector (Cartharius et al. 2005).

\section{Reporter plasmids construction}

The promoter fragment of zebrafish cyp11a1 was cloned into pGL3-Basic luciferase reporter plasmid (Promega) and pEGFP-1 reporter plasmid (Clontech). It was also used as a template to generate the $1 \cdot 5,1$, and $0.5 \mathrm{~kb}$ fragments using primers containing flanking KpnI and BglII restriction sites, and subsequently cloned into pGL3-Basic vector. The proximal and FREd were mutagenized separately, or simultaneously using the Quickchange site directed mutagenesis kit (Stratagene, La Jolla, CA, USA) with the following primers, 5'-CCTTCCTTTAGCATGTTGACCTCGGTGAAAGTAAATGCGGATC-3' for FREd and 5'-CCTTTAGTAGTTCATTCACCGTGACCTGTGTGGTGTTTTGGC-3' for FREp.

\section{Cell culture}

The mouse L $\beta$ T2 gonadotrope and human HepG2 hepatoma cell lines were maintained in DMEM supplemented with $10 \%$ fetal bovine serum (FBS), $2 \mathrm{mM}$ L-glutamine, and $1 \mathrm{mM}$ sodium pyruvate. The mouse MA-10 Leydig tumor cell line was maintained in Waymouth's medium supplemented with $15 \%$ horse

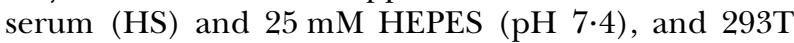
monkey kidney cells were maintained in RPMI medium supplemented with $10 \%$ FBS. The mouse Y1 adrenocortical and Chinese hamster ovary CHO-K1 cell lines were maintained in Ham's F12K medium supplemented with $10 \%$ HS and $2.5 \%$ FBS, and $10 \%$ FBS respectively. All cells were maintained at $37^{\circ} \mathrm{C}$ in $5 \%$ $\mathrm{CO}_{2}$ with penicillin $(100 \mathrm{U} / \mathrm{ml})$ and streptomycin $(100 \mu \mathrm{g} / \mathrm{ml})$ added into the culture media. Cells were grown to $80-90 \%$ confluency prior to seeding for transfections or routine passages. All basal media were purchased from Invitrogen. 


\section{Transient transfection and dual-luciferase reporter assay}

On the day before transfection, cells were plated in 24-well plates with a density of $0 \cdot 8-1.5 \times 10^{5}$ cells per well. Transient transfections were carried out using FUGENE 6 transfection reagent (Roche) except for MA-10 cells, which were transfected using Lipofectamine 2000 (Invitrogen). Cells were transfected with $0.4 \mu \mathrm{g}$ each luciferase reporter plasmid and $2 \mathrm{ng}$ pRL-SV40 as a transfection efficiency control. After $24 \mathrm{~h}$, cells were harvested and lysed in $1 \times$ passive lysis buffer provided in the dual Luciferase reporter assay system (Promega). Luciferase activity in $20 \mu \mathrm{l}$ homogenate was assayed using a Lumat LB9507 luminometer (Berthold Technologies, Bad Wilbad, Germany). The firefly luciferase activity conferred by the respective cyp11a1-luc constructs was normalized by the Renilla luciferase activity conferred by pRL-SV40. Data are presented as the mean \pm s.E.M. of results from four independent transfections. The transfections were repeated at least once to ensure the reproducibility of the data.

\section{Electrophoretic mobility shift assay}

Fflb proteins were produced by in vitro translation from pcDNA3.1ff1b (Liu et al. 2003) using the TNT T7 quick coupled transcription/translation system (Promega). To verify the identity of Fflb protein, the translated proteins were biotinylated by adding Transcend tRNAs (Promega) to the coupled transcriptiontranslation reaction and the presence of a $50 \mathrm{kDa}$ band, representing the Ff1b protein, was confirmed by chemiluminescence. EMSA was performed using the LightShift Chemiluminescent EMSA kit (Pierce, Rockford, IL, USA). Oligonucleotides containing the FREd (5'-GTTGACCTTGGGAAAAGTAAATG- $\left.3^{\prime}\right)$, FREp (5'-GTAGTTCATTGTCCTT-GACCTGTG-3' ${ }^{\prime}$, the corresponding mutants, FREdMut (5'-GTTGACCTCGGTGAAAGTA-AATG-3'), and FREpMut (5'-GTAGTTCATTCACCGTGACCTGTG-3') were synthesized and labeled using the Biotin $3^{\prime}$ end DNA labeling kit (Pierce). For each EMSA reaction, $8 \mu$ in vitro translated mixture was mixed with $20 \mathrm{fmol}$ biotin-labeled DNA probes and $1 \mu \mathrm{g}$ Poly $\mathrm{dI} \times \mathrm{dC}$ in $1 \times$ binding buffer (10 mM Tris, $100 \mathrm{mM} \mathrm{KCl,} 1 \mathrm{mM}$ dithiothreitol; pH $7 \cdot 5)$ in a total reaction volume of $20 \mu \mathrm{l}$ and incubatd at $25^{\circ} \mathrm{C}$ for $20 \mathrm{~min}$. Unbound DNA probes were resolved from protein-DNA complexes by electrophoresis on a $5 \%$ polyacrylamide gel. After electrophoresis, DNA oligonucleotides were transferred onto a $\zeta$-probe membrane (Bio-Rad) by electroblotting and u.v. crosslinked. Biotin-labeled DNAs were visualized with streptavidin-bound HRP and Luminol/Enhancer chemiluminescent substrate (Pierce) and chemiluminescence detected by exposure to X-ray film (Fujifilm, Tokyo, Japan).

\section{Chromatin immunoprecipitation}

ChIP assays were performed using the ChIP assay kit (Upstate Biotechnology, Lake Placid, NY, USA). Ovarian cell suspension was prepared from freshly dissected ovary tissue of adult zebrafish in $1 \times$ PBS. Cells were fixed with $1 \%$ formaldehyde for $15 \mathrm{~min}$, washed with chilled $1 \times$ PBS, and resuspended in $200 \mu \mathrm{l}$ SDS lysis buffer. Following lysis for $10 \mathrm{~min}$ on ice, lysate was sonicated 14 times for $10 \mathrm{~s}$ separated by a 4-min interval, at $20 \%$ of maximum amplitude using the Vibra Cell sonicator VC 505 (Sonics and Materials Inc., Newton, CT, USA) equipped with a $3 \mathrm{~mm}$ tip. After sonication, one-tenth of supernatant was removed as input and the remaining was diluted 10 -fold prior to immunoclearing by $75 \mu \mathrm{l}$ salmon sperm DNA/protein A agarose-50\% slurry for $30 \mathrm{~min}$ at $4{ }^{\circ} \mathrm{C}$. Immunoprecipitation was performed overnight at $4{ }^{\circ} \mathrm{C}$ with $5 \mu \mathrm{g}$ anti-Ff $1 \mathrm{~b}$ antisera \#1660 (Biogenes, Berlin, Germany). The region that the Fflb antibody recognizes (CAYLHQEQSGRGKLE) is unique to Fflb and the Fflb antibody does not cross-react with other Ff1 isoforms in Western blotting (data not shown). The pre-immune serum (PIS) from the same rabbit was used as negative control. Immunoprecipitated agarose was sequentially washed for $4 \mathrm{~min}$ each in $1 \mathrm{ml}$ low-salt, high-salt, and lithium chloride immune complex wash buffers, and finally washed twice with Tris/EDTA buffer. Histone complexes were finally eluted from the antisera by freshly prepared elution buffer ( $1 \%$ SDS, $0 \cdot 1 \mathrm{M} \mathrm{NaHCO} 3$ ). Cross-linking of histone-DNA (including the input samples) was reversed by $5 \mathrm{M} \mathrm{NaCl}$ at $65^{\circ} \mathrm{C}$ for $4 \mathrm{~h}$. DNA fragments were extracted by phenol/chloroform extraction and ethanol precipitation with the addition of glycogen $(20 \mu \mathrm{g} / \mu \mathrm{l})$.

An aliquot of the extracted DNA $(1 \mu \mathrm{l})$ was used for PCR analysis. A pre-denaturation of 5 min at $95^{\circ} \mathrm{C}$ was followed by 30 cycles $\left(30 \mathrm{~s}\right.$ at $95{ }^{\circ} \mathrm{C}, 30 \mathrm{~s}$ at $56^{\circ} \mathrm{C}$, and $1 \mathrm{~min}$ at $72^{\circ} \mathrm{C}$ ), and a final elongation of $10 \mathrm{~min}$ at $72^{\circ} \mathrm{C}$. To detect enrichment in the proximity of FREd, primers spanning -1669 and -1501 were used; FREdChIPF, 5'-CGGTGTGAAGCAATATGCAT-3' ${ }^{\prime}$, and FREdChIPR, 5'-CCAAGGTCAACATGCTAAAGG-3' ${ }^{\prime}$. For FREp, primers spanning -249 and -1 were used; FREpChIPF, 5'-CGGATGTGTTTGATTCAAGG-3' ${ }^{\prime}$, and FREpChIPR， 5'-GGCACTCTCTCTCTCTACTTC-3'. As negative control, primers spanning from -471 to -255 region of the zebrafish keratin 8 promoter, where no consensus FRE is present, were included in the PCR analysis; keratin8F, 5'-GCGCCCCACCCAGATAAAA-3', 
and keratin8R, 5'-ATGTTGTCACACCCAGCCTG-3'. PCR reactions were resolved on $1.5 \%$ agarose gel and visualized by SYBRSafe staining.

\section{Microinjection of EGPF and luciferase reporter plas- mids into zebrafish embryos}

The cyp11a1 promoter-EGFP plasmid $(100 \mathrm{ng} / \mu \mathrm{l})$ was diluted in $1 \times$ Danieau's buffer and $0.1 \%$ phenol red. Microinjection was carried out under a dissection microscope using a Nanoliter 2000 microinjector (WPI). One- to two-cell stage zebrafish embryos were microinjected with $2 \cdot 3 \mathrm{nl}$ plasmid solution. Embryos were monitored for GFP expression under u.v. microscope (Zeiss Axiovert 25) from 28 hours post fertilization (hpf) onwards. EGFP fluorescence was subsequently captured using confocal microscope (Olympus IX70) equipped with FV300 imaging software.

To quantify the cyp11a1 promoter activity, zebrafish embryos were injected with $4.6 \mathrm{nl}$ DNA solution containing $50 \mathrm{ng} / \mu \mathrm{l}$ of the respective CYP11A1 promoter-luciferase constructs, $5 \mathrm{ng} / \mu \mathrm{l}$ internal control, pRL-CMV, and $100 \mathrm{ng} / \mu \mathrm{l} \mathrm{pcDNA3.1fflb} \mathrm{(Liu} \mathrm{et} \mathrm{al.}$ 2003). Embryos were checked $4 \mathrm{~h}$ later and only those that were healthy and showed strong phenol red staining were retained for luciferase assay. Embryos were harvested and dechorionated at $14 \mathrm{hpf}$. Embryo extract was prepared from 25 embryos by pipetting up and down in $20 \mu \mathrm{l}$ of $1 \times$ passive lysis buffer and luciferase activity was determined using $20 \mu \mathrm{l}$ homogenate.

\section{Results}

\section{Conserved cis-acting elements are identified in zebrafish cyp11a1 promoter}

A $1.7 \mathrm{~kb} \mathrm{5'-flanking} \mathrm{region} \mathrm{of} \mathrm{the} \mathrm{zebrafish} \mathrm{cyp11a1}$ was recovered by genome walking and verified by DNA sequencing. The putative promoter sequence matched 158905-160637 of chromosome 25 (contig AL929050.6; Zebrafish Ensembl genome database, $\mathrm{Zv} 7$ ). Two TATA boxes were located in the proximity of the FREp but the transcription start site has yet to be determined experimentally. When the promoter sequence was analyzed for potential TFBSs using MatInspector, binding sites for Ff1b, AP-1, Sp1, and factors that bind cAMP response elements (CREs) were evident, and were conserved when compared with pufferfish Tetraodon nigroviridis, mouse, and human (Fig. 1). These factors have been previously implicated in the transcriptional regulation of CYP11A1 promoter (Guo et al. 2007). Besides, several bindings sites for known co-regulators of SF1 including Sox proteins,
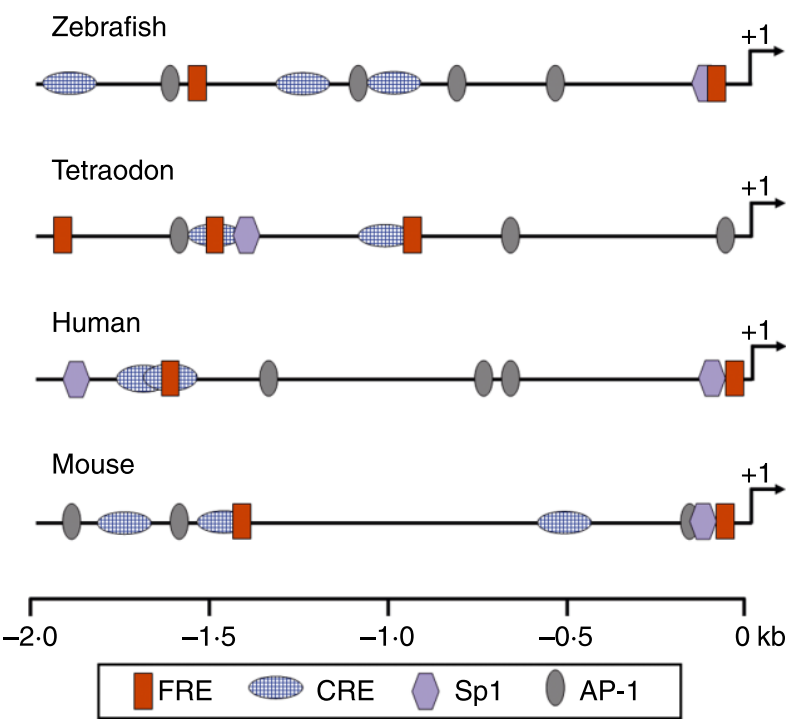

Figure 1 Conservation of cis-elements predicted in the $5^{\prime}$ flanking promoter region of zebrafish CYP11A1 in comparison with the equivalent $2 \mathrm{~kb}$ region in the gene promoter of tetraodon, human, and mouse. Binding sites of the transcription factors indicated were predicted using the web-based MatInspector program. Only binding sites for transcription factors that have previously been implicated in the transcriptional regulation of CYP11A1 promoter were shown. Full colour version of this figure available via http:// dx.doi.org/10.1677/JME-09-0029.

GATA factors, and WT1 have also been identified in these promoters (data not shown). At least two CREs were mapped for all species. Importantly, the two identified FREs were highly conserved across zebrafish, human, and mouse.

\section{The $1.7 \mathrm{~kb}$ cyp11a1 promoter is transcriptionally active in different cell lines}

We evaluated the ability of the putative cypl1a1 promoter to activate luciferase activity in cell lines derived from steroidogenic and non-steroidogenic lineages. In $\mathrm{Y} 1$ adrenocortical cells, luciferase activity was $12 \cdot 2$-fold above the basal level conferred by pGL3Basic (Fig. 2). In CHO-K1 ovarian, MA-10 testis, and L $\beta$ T2 cells, luciferase activity was increased by $4 \cdot 5-, 6 \cdot 7$ and $3 \cdot 5$-fold respectively. Intriguingly, luciferase activity was increased by 18 -fold in the non-steroidogenic 293T embryonic kidney cells, but was, however, inactive in HepG2 liver carcinoma cells. These results indicate that the regulatory cis-elements within this cyp11a1 promoter are sufficient to activate gene expression in steroidogenic cell lines. Subsequent transfection studies were performed in Y1 cells, as the adrenal cortex represents one of the major tissues where cyp11a1 is endogenously expressed and, thus, should provide the best cellular niche for the investigation of the zebrafish cyp11a1 promoter activity. 


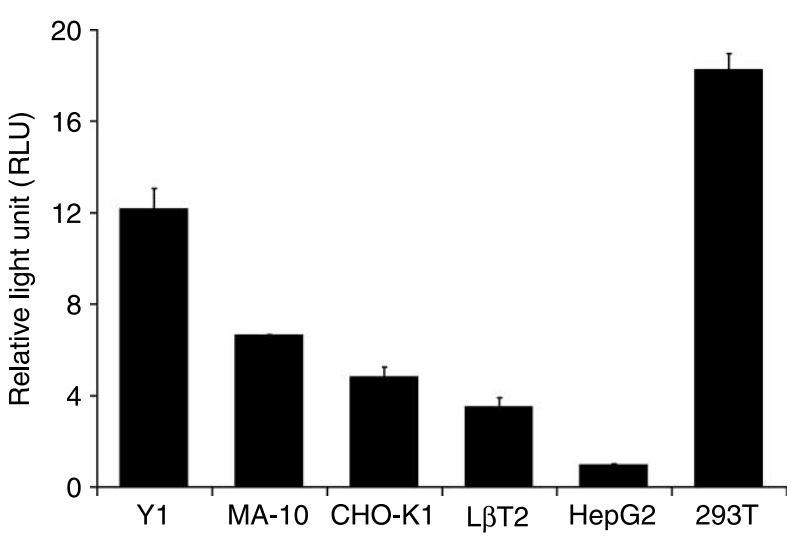

Figure 2 Promoter activity of the zebrafish cyp11a1 promoter in cell lines from different lineages. The $1.7 \mathrm{~kb}$ zebrafish CYP11A1 promoter was cloned upstream of the luciferase gene in pGL3Basic vector, and transfected into $\mathrm{Y} 1$ (adrenocortical), MA-10 (Leydig), CHO-K1 (ovarian), L $\beta T 2$ (gonadotrope), HepG2(hepatoma), and 293T (kidney) cell lines. Renilla luciferase expression vector, pRL-SV40, was co-transfected for normalization of transfection efficiency. Data are expressed as normalized luciferase activity in relative light unit (RLU) measured from cells transfected with cyp11a1-luc or promoterless pGL3Basic vector (adjusted to 1.0) from one representative experiment, and represent the mean of luciferase activity \pm S.E.M. $(n=4)$.

\section{FREp is the major mediator of cyp11a1 promoter activity}

Various $5^{\prime}$ deleted fragments of the $1.7 \mathrm{~kb}$ promoter were generated by PCR and cloned into pGL3-Basic for the assessment of their activities. Luciferase assay revealed that the truncation of this putative cyp11a1 promoter down to $1 \mathrm{~kb}$ did not affect luciferease activity significantly (Fig. 3). Even when FREd was truncated, as in both the 1.5 and $1 \mathrm{~kb}$ promoter fragments, luciferase activity was comparable with that of $1.7 \mathrm{~kb}$ fragment. Only when the promoter was truncated down to $500 \mathrm{bp}$, the luciferase activity was reduced to $44 \cdot 4 \%$. Nevertheless, the $500 \mathrm{bp}$ promoter was still functional, probably due to the intact FREp.

To further explore their functions, we mutagenized the FREd and FREp individually or in combination, such that three nucleotides within the core or flanking recognition site were mutagenized discontinuously in reminiscent of the mutagenesis performed in human CYP11A1 promoter (Hu et al. 2001, Hsu et al. 2004; Fig. 4). Transient transfections into Y1 cells showed that mutation in the FREp reduced luciferase activity by $89.6 \%$, while the mutation in FREd only marginally reduced the luciferase activity by $11.6 \%$. When both FREp and FREd were mutated it rendered the cyp11a1 promoter completely inactive. The results demonstrate that the FREp and FREd do not have similar effects in gene activation. In agreement with the findings from human CYP11A1 promoter, the FREp, which is situated in the basal promoter, was found to be the major regulator for the promoter activity in vitro, whereas the function of the FREd in basal gene expression is less obvious (Hu et al. 2001, Hsu et al. 2004).

\section{Ff1b binds to both FREd and FREp in vitro}

To determine if Fflb binds to the two FREs selectively, oligonucleotides containing the FREd and the FREp were synthesized, biotin-labeled, and EMSA was carried out. Fflb proteins were transcribed and translated in vitro from the pcDNA3.1ff1b overexpression plasmid. The identity of Fflb, which corresponds to a $\sim 50 \mathrm{kDa}$ band in a SDS-PAGE, was verified by biotin-labeling and immunoblotting (data not shown). As shown in Fig. 4, no band shift was observed when the biotin-labeled oligonucleotides were incubated with reaction mixtures using the pcDNA3.1 as template (lanes 1 and 6). Mobility complexes were only observed when the reaction mixtures containing $\mathrm{Ffl}$ b proteins were used (lanes 2 and 7). Fflb proteins, however, did not bind the mutated version of the biotin-labeled FREd and FREp (lanes 3 and 8). The Fflb-FRE complexes were effectively competed by a 200 -fold molar excess of the corresponding unlabeled FREs (lanes 4 and 9). The Fflb-FRE complexes were, however, unaffected by a

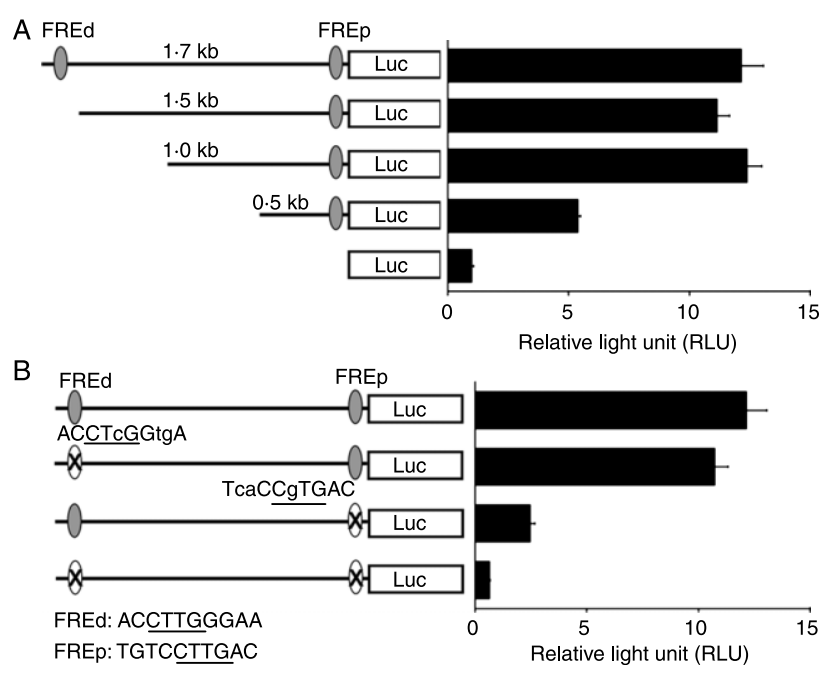

Figure 3 The proximal FRE is crucial for the activation of the zebrafish cyp11a1 promoter. (A) Transcriptional activity of various truncated cyp11a1 promoter fragments was measured by luciferase assay in $Y 1$ cells. The relative positions and nucleotide sequences of distal $\mathrm{Ff} 1 \mathrm{~b}$ response element (FREd) and proximal $\mathrm{Ff} 1 \mathrm{~b}$ response element (FREp) are indicated. (B) The two FREs were site-directed mutagenized either individually or simultaneously, with mutated residues indicated in lower case. Transcriptional activities of the mutagenized promoters were assessed in Y1 cells. Renilla luciferase expression vector, pRL-SV40, was co-transfected for normalization of transfection efficiency. Data are expressed as normalized luciferase activity in relative light unit from one representative experiment, and represent the mean of luciferase activity \pm S.E.M. $(n=4)$. 


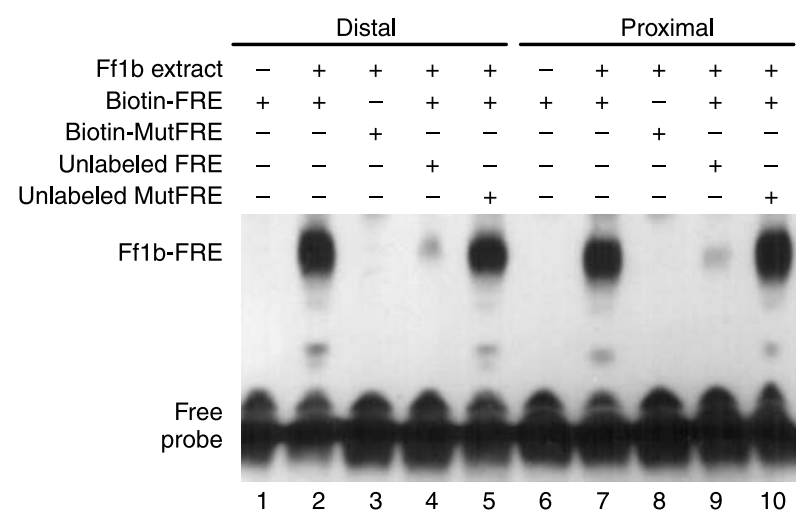

Figure $4 \mathrm{Ff} 1 \mathrm{~b}$ binds to both the distal and proximal FRE (FREd and FREp) in electrophoretic mobility shift assay. Biotin-labeled wild-type or mutated oligonucleotides (30 bp) of FREd or FREp were incubated with rabbit reticulocyte in which pcDNA3.1 vector (lanes 1 and 6) or ff1b overexpression plasmid, pcDNA3.1ff1b, (lanes 2-5 and 7-10) was used as template for in vitro transcription and translation. For competition assay, $\sim 200$-fold molar excess of the respective unlabeled FRE or mutated FRE was used.

200-fold molar excess of the unlabeled mutated FREs (lanes 5 and 10). These observations demonstrated the specificity of Fflb binding to both FREs.

Following the discovery that Fflb binds to both the FREd and FREp, we used EMSA competitive binding assays to determine the relative binding affinity of Fflb to both the FREs. The binding of Fflb to biotin-labeled

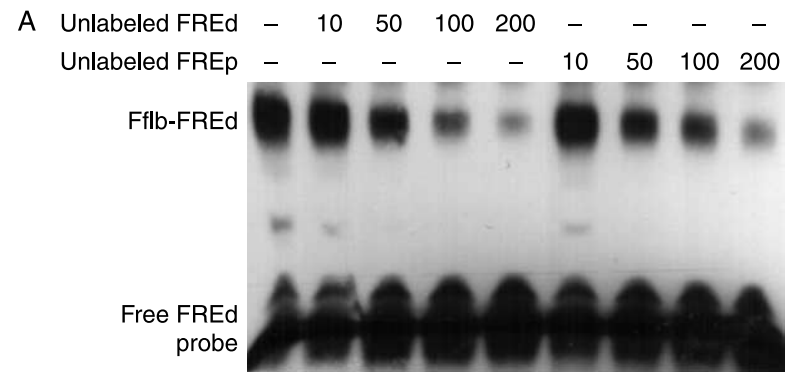

B Unlabeled FREd - $\begin{array}{llllllllll}10 & 50 & 100 & 200 & - & - & - & -\end{array}$

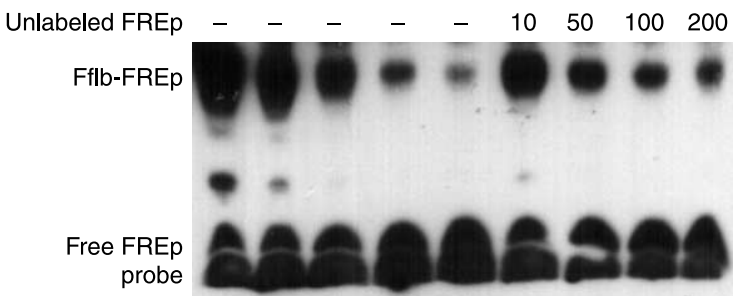

Figure 5 Competitive binding of $F f 1 b$ to the FREd and FREp in electrophoretic mobility shift assay. (A) Biotin-labeled FREd or (B) FREp oligonucleotides (30 bp) were incubated with rabbit reticulocyte in which $f f 1 b$ overexpression construct, pcDNA3.1ff1b, was used as template for in vitro transcription and translation. For competition, 10-, 50-, 100-, and 200-fold molar excess of the respective unlabeled FRE were used.
FREd (Fig. 5A) and to biotin-labeled FREp (Fig. 5B) was progressively reduced by increasing concentrations of unlabeled FREp and FREd at 10-, 50-, 100-, and 200-fold molar excesses. The difference in binding affinity of Ff1b to FREd and FREp, was however, only marginal (Fig. 5).

\section{Ff1b binds to both FREd and FREp in vivo}

ChIP assay was carried out to determine if Fflb occupied the two FREs in vivo. Chromatin was immunoprecipitated by Fflb antiserum from ovarian tissues, where $\mathrm{Fflb}$ is abundantly expressed. In agreement with the EMSA results, Fflb bound to both FREd and FREp in vivo as shown by the enrichment of the two promoter regions in PCR analyses following ChIP (Fig. 6). This enrichment did not take place when the PIS was used instead of Fflb antiserum. Likewise, the enrichment was not seen on keratins promoter where no consensus FRE was present. This is the first in vivo demonstration of the association of $\mathrm{Fflb}$ to one of its target gene promoter.

\section{The cyp11a1 promoter targets EGFP specifically to the interrenal and genital ridge}

To investigate if the $1.7 \mathrm{~kb}$ fragment was sufficient to direct the tissue-specific expression of CYP11A1 in zebrafish, it was cloned into pEGFP-1 reporter vector. When the cyp11a1-EGFP plasmid was microinjected into zebrafish embryos at $1-2$ cell stages, the $1.7 \mathrm{~kb}$ promoter was able to target EGFP expression specifically to the interrenal gland and genital ridge (Fig. 7A). Nearly $10 \%$ of the microinjected embryos showed EGFP fluorescence at these tissues from $\sim 32$ hpf onwards.

To quantify the promoter activity and to assess its responsiveness to $f f 1 b$ overexpression in zebrafish, the cyp11a1-luc reporter plasmid was microinjected into

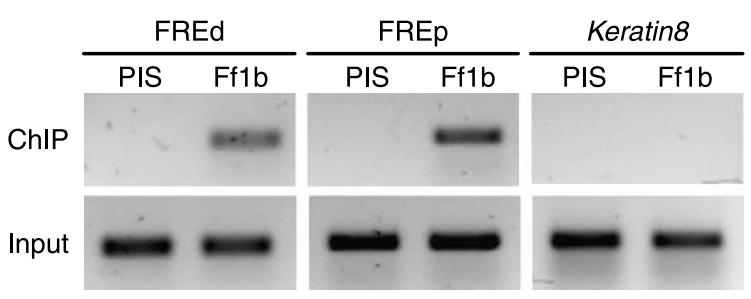

Figure $6 \mathrm{Ff} 1 \mathrm{~b}$ binds to both distal and proximal FREs in vivo as revealed by ChIP. Chromatin was prepared from freshly dissected zebrafish ovary and immunoprecipitated with antiserum against Ff1b or pre-immume serum (PIS) from the same rabbit. Genomic DNA extracted was analyzed by PCR using primers covering the nucleotide positions; -1669 and -1501 for FREd, -249 and -1 for FREp, and from -471 to -255 for keratin8 promoter, which does not contain any consensus FRE. 
A
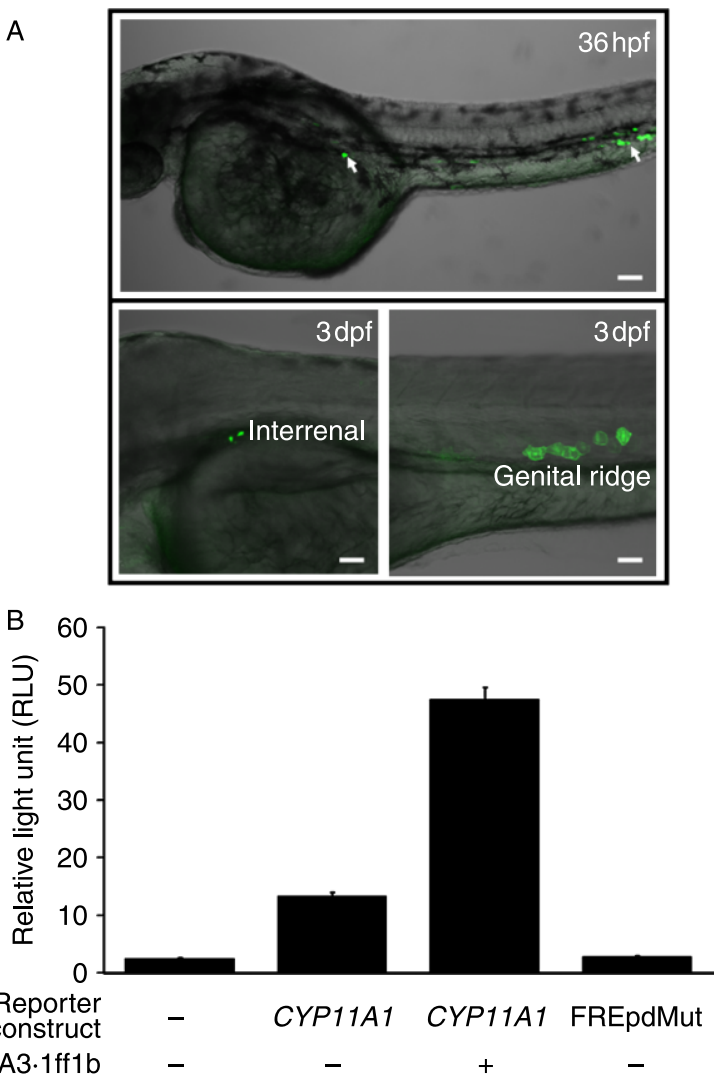

pcDNA3.1ff1b

Figure 7 The $1.7 \mathrm{~kb}$ zebrafish cyp11a1 promoter directs tissuespecific expression of EGFP in zebrafish embryos and overexpression of Ff1b enhances its promoter activity. (A) The zebrafish cyp11a1-pEGFP construct was microinjected into zebrafish embryos at 1- to 2-cell stages. EGFP fluorescence was captured by confocal microscope equipped with a $488 \mathrm{~nm}$ laser.

(B) Embryos at the 1- to 2-cell stages were injected with the different combinations of reporter and ff1b-expression plasmids. pRL-CMV was included as a normalization control for firefly luciferase activity. Embryos $(n=25)$ were harvested at $14 \mathrm{hpf}$ for determination of luciferase activity. Data are expressed as normalized luciferase activity measured from one representative microinjection experiment \pm S.E.M. $(n=4)$. Full colour version of this figure available via http://dx.doi.org/10.1677/JME-09-0029.

zebrafish embryos and transactivation assay was carried out as previously described (Liu et al. 2003). As shown in Fig. $7 \mathrm{~B}$, the $1.7 \mathrm{~kb}$ zebrafish cyp11a1 promoter was activated by $4 \cdot 4$-fold as compared with the promoterless pGL3-Basic vector. When the $f f 1 b$ overexpression plasmid, pcDNA3.1ff1b, was co-injected, the luciferase activity driven by the cyp11al-luc was further enhanced by $3 \cdot 4$-fold. When both the FREs were mutated, the promoter construct (FREpdMut) was completely inactive. Taken together these findings provide the first in vivo evidence that Fflb potentiates the activation of the zebrafish cyp11a1 promoter and that a $1.7 \mathrm{~kb}$ promoter fragment is sufficient to activate tissue-specific expression in interrenal gland and genital ridge.

\section{Discussion}

This study reports the cloning and functional characterization of a $1.7 \mathrm{~kb}$ promoter fragment of zebrafish cyp11a1. Bioinformatics analysis revealed conserved modules of cis-elements within the cyp11a1 promoter in comparison with other species including tetraodon, human, and mouse. By transient transfection studies, we showed that the $1.7 \mathrm{~kb}$ promoter was capable of activating luciferase activity in a variety of steroidogenic cell lines and that the transcriptional activation was largely dependent on an intact FREp. Our EMSA and ChIP results confirmed the binding of Fflb to both FREd and FREp. Most importantly, the $1.7 \mathrm{~kb}$ promoter could direct tissue-specific expression of EGFP in zebrafish embryos in a developmentally regulated fashion and the overexpression of Fflb further potentiated the activity of the cyplla1 promoter in vivo.

From the study of the human CYP11A1 promoter, many transcription factors, including AP-1, SF1, Sp1, TReP-132, AP-2, NF-1, and Ets, have been identified to be important for its transcriptional activation (Guo et al. 2007). In the zebrafish cypl1a1 promoter, only binding sites for SF1, Sp1, and AP-1 have been mapped with confidence. Nevertheless, the presence of the two highly conserved FREs is consistent with the role of SF1 acting as a central regulator of the transcriptional activation of steroid hydroxylases. The identification of CRE and binding sites for transcription factors that have been implicated in SF1 transcriptional activity or in the activation of CYP11A1 promoter, like Sox proteins (De Santa et al. 1998), GATA factors (Tremblay \& Viger 1999), WT-1 (Shen et al. 1994), and AP-1 (Li et al. 1999, Huang et al. 2001), within the $1.7 \mathrm{~kb}$ promoter indicates a conserved mode of transcriptional regulation across different species. The importance of these transcription factors on the activation of the zebrafish cyp11a1 promoter, however, needs to be further evaluated.

Despite the conservation of cis-acting elements within the $1.7 \mathrm{~kb}$ promoter region, the zebrafish and human CYP11A1 promoters are not functionally equivalent. In $\mathrm{Y} 1$ cells, the $1.7 \mathrm{~kb}$ human promoter was transcriptionally more active ( $\sim 70 \%$ higher; data not shown) than the zebrafish promoter, but it was completely inactive when microinjected into zebrafish embryos as measured by luciferase and EGFP reporter activity (data not shown). This is intriguing considering that the same $1.7 \mathrm{~kb}$ human promoter could direct LacZ expression specifically to the adrenals and gonads in transgenic mice (Hu et al. 1999, 2001). In zebrafish, cyp1 1a1 expression has also been detected in the brain and yolk syncytial layer (Hsu et al. 2002, 2006). Nevertheless, the $1.7 \mathrm{~kb}$ cyp11a1 promoter does not direct EGFP expression to these tissues in our transient 
transgenesis studies in zebrafish embryos. These expression domains are likely to be independent of Ff1b regulation and the cis-elements responsible are likely to lie outside the $1.7 \mathrm{~kb}$ promoter. Indeed, the expression of Cyp11a1 for rat in the central and peripheral nervous system has been shown to be independent of SF1, as $S f 1$ is not expressed in these tissues (Zhang et al. 1995, Hammer et al. 2004).

Not unexpectedly, the $1.7 \mathrm{~kb}$ zebrafish cyp11a1 promoter is transcriptionally active in steroidogenic cell lines like Y1, MA-10, and CHO-K1, and in L $\beta \mathrm{T} 2$, which originated from pituitary gonadotropes. This is attributed to the presence of SF1, which replaces Fflb in the activation of the zebrafish cypl1al promoter. However, the high levels of cyplla1 promoter activity in 293T cells, which is of kidney origin, is surprising and indicates the presence of another trans-acting factor that is capable of assuming the functional role of SF1. This is not new for the transcriptional regulation of CYP11A1 expression. In placenta tissue, which does not express SF1, AP-2 assumes the role of activating the transcription of CYP11A1 (Ben Zimra et al. 2002). Intriguingly, the endogenous presence of LRH1 in HepG2 cells (Baiz et al. 2009), which is a paralogue of SF1 and binds the same FRE (Fayard et al. 2004), did not activate the zebrafish cyplla1 promoter. This observation, once again, has highlighted the potential roles of other transcription factors that interact with SF1 or Fflb in regulating the tissue-specific activity of the cyp11a1 promoter.

Why is it necessary to have two FREs in the promoter of cyp11a1? Previous studies on the human promoter have pointed to an explanation that while FREp is responsible for the basal activity, FREd is responsible for hormonal regulation and tissue selectivity (Guo et al. 2007). This difference in functionality of the two FREs is due to the different cis-acting elements associated with individual FREs. The same feature is seen in the zebrafish cypllal promoter, where FREd is surrounded by a CRE and binding sites for AP-1 and GATA factors, while FREp is surrounded by TATA boxes and binding sites for transcription factors such as Sox and Wt-1. Transfection studies involving DNA constructs carrying different combinations of the mutated FREs have ascertained the pivotal role of the FREp in the basal activity of cyp11a1 promoter. Thus, at the proximal promoter, Ff1b bound to FREp regulates the basal promoter activity by interacting with transcription factors in the Pol II initiation complex such as TFIIB, CBP/p300, and AP-1 (Monte et al. 1998, Li et al. 1999). Although the decrease in promoter activity is marginal following the truncation and mutation of FREd, the results are indicative of its possible role in basal transcription of the cyp11a1 promoter.
Despite the stronger influence of the FREp mutation on basal promoter activity, EMSA and ChIP assays showed that Fflb binds to both the distal and FREp in vitro and in vivo. Differential binding of SF1 to its two binding sites has been demonstrated in the human and rat CYP11A1 promoters (Clemens et al. 1994, Hu et al. 2001). This difference in the functionality of the two FREs has added one more layer of complexity in Fflb transcriptional activity. It will be interesting to dissect the molecular events controlling Fflb binding to the two FREs in terms of hormonal regulation and to check whether this is related to the dosage-dependent effect. Although our current data suggests Fflb as the predominant regulator of cyp11a1 promoter, they do not preclude the possible involvement of other Ff1 isoforms that can potentially bind the FREs, particularly if they are co-expressed in the same tissues.

The ability of the zebrafish cyp11a1 promoter to specifically target EGFP expression to the interrenal gland and genital ridge is reminiscent of the human CYP11A1, where a $1.7 \mathrm{~kb}$ promoter is sufficient to direct LacZ expression to adrenals and gonads in transgenic mice (Hu et al. 2001). The low percentage of embryos ( $\sim 10 \%$ ) expressing EGFP at the two tissues, however, might be indicative that the $1.7 \mathrm{~kb}$ promoter is relatively weak, and additional cis-elements outside of this fragment might be important to fully recapitulate the endogenous expression level. The characterization of additional $5^{\prime}$ flanking sequence would certainly be important. More importantly, by combining microinjection and transient transactivation assays in zebrafish embryos, we provided the first in vivo evidence on the responsiveness of the $1.7 \mathrm{~kb}$ cyp11a1 promoter to Fflb, indicating that this is a viable approach to study the activation of other promoters that are dependent on Fflb.

In conclusion, a $1.7 \mathrm{~kb}$ promoter region of zebrafish cyp11a1 has been characterized in terms of its basal transcriptional activity and its regulation by Fflb. The direct influence of Fflb on the activity of zebrafish cyp11a1 promoter highlighted how the transcriptional regulation could be conserved even after 400 million years of evolution (Kumar \& Hedges 1998). Our findings also ascertained the orthology of Fflb to its mammalian counterpart SF1, although it remains to be seen if ffld, which is also an ortholog of SF1 (von Hofsten et al. 2005, Kuo et al. 2005), also retains the ability to regulate the expression of cyp11a1 and other FF1b responsive genes.

\section{Declaration of interest}

We declare that there is no conflict of interest that could be perceived as prejudicing the impartiality of the research reported. 


\section{Funding}

This work was supported by the National University of Singapore and a BMRC grant (BMRC07/1/21/19/527) from A*STAR. S I was supported by a NUS Postgraduate Research Scholarship.

\section{Acknowledgements}

We thank Prof. Chung Bon-chu (Institute of Molecular Biology, Academia Sinica, Taiwan) for her generosity in sharing her unpublished data and the human CYP11A1 promoter constructs with us. We also like to thank Professors Martin Lee (Department of Physiology, NUS) and Pamela Mellon (UCSD) for their kindness in sharing the MA-10 and L $\beta$ T2 cell lines with us respectively.

\section{References}

Ahlgren R, Suske G, Waterman MR \& Lund J 1999 Role of Sp1 in cAMP-dependent transcriptional regulation of the bovine CYP11A gene. Journal of Biological Chemistry 274 19422-19428.

Baiz D, Pozzato G, Dapas B, Farra R, Scaggiante B, Grassi M, Uxa L, Giansante C, Zennaro C, Guarnieri G et al. 2009 Bortezomib arrests the proliferation of hepatocellular carcinoma cells HepG2 and JHH6 by differentially affecting E2F1, p21 and p27 levels. Biochimie 91 373-382.

Bakke M, Zhao L, Hanley NA \& Parker KL 2001 SF-1: a critical mediator of steroidogenesis. Molecular and Cellular Endocrinology $1715-7$.

Bury NR \& Sturm A 2007 Evolution of the corticosteroid receptor signalling pathway in fish. General and Comparative Endocrinology $15347-56$.

Cartharius K, Frech K, Grote K, Klocke B, Haltmeier M, Klingenhoff A, Frisch M, Bayerlein M \& Werner T 2005 MatInspector and beyond: promoter analysis based on transcription factor binding sites. Bioinformatics 21 2933-2942.

Chai C \& Chan WK 2000 Developmental expression of a novel Ftz-F1 homologue, fflb (NR5A4), in the zebrafish Danio rerio. Mechanisms of Development 91 421-426.

Chai C, Liu YW \& Chan WK 2003 Fflb is required for the development of steroidogenic component of the zebrafish interrenal organ. Developmental Biology 260 226-244.

Chung BC, Guo IC \& Chou SJ 1997 Transcriptional regulation of the CYP11A1 and ferredoxin genes. Steroids 62 37-42.

Clemens JW, Lala DS, Parker KL \& Richards JS 1994 Steroidogenic factor-1 binding and transcriptional activity of the cholesterol side-chain cleavage promoter in rat granulosa cells. Endocrinology 134 1499-1508.

Fayard E, Auwerx J \& Schoonjans K 2004 LRH-1: an orphan nuclear receptor involved in development, metabolism and steroidogenesis. Trends in Cell Biology 14 250-260.

Gallo VP \& Civinini A 2003 Survey of the adrenal homolog in teleosts. International Review of Cytology 230 89-187.

Guo IC, Hu MC \& Chung BC 2003 Transcriptional regulation of CYP11A1. Journal of Biomedical Science 10 593-598.

Guo IC, Shih MC, Lan HC, Hsu NC, Hu MC \& Chung BC 2007 Transcriptional regulation of human CYP11A1 in gonads and adrenals. Journal of Biomedical Science 14 509-515.

Hammer GD \& Ingraham HA 1999 Steroidogenic factor-1: its role in endocrine organ development and differentiation. Frontiers in Neuroendocrinology 20 199-223.

Hammer F, Compagnone NA, Vigne JL, Bair SR \& Mellon SH 2004 Transcriptional regulation of $\mathrm{P} 450 \mathrm{scc}$ gene expression in the embryonic rodent nervous system. Endocrinology 145 901-912. von Hofsten J, Larsson A \& Olsson PE 2005 Novel steroidogenic factor1 homolog (ffld) is coexpressed with anti-Mullerian hormone (AMH) in zebrafish. Developmental Dynamics 233 595-604.

Hsu HJ, Hsiao P, Kuo MW \& Chung BC 2002 Expression of zebrafish cyp1la1 as a maternal transcript and in yolk syncytial layer. Gene Expression Patterns 2 219-222.

Hsu HJ, Lin G \& Chung BC 2003 Parallel early development of zebrafish interrenal glands and pronephros: differential control by wt1 and fflb. Development 130 2107-2116.

Hsu NC, Shih MC \& Chung BC 2004 Study of the function of proximal SF-1 binding sites on Cyp11A1 promoter. Endocrine Research 30 813-814.

Hsu HJ, Liang MR, Chen CT \& Chung BC 2006 Pregnenolone stabilizes microtubules and promotes zebrafish embryonic cell movement. Nature 439 480-483.

Hu MC, Chou SJ, Huang YY, Hsu NC, Li H \& Chung BC 1999 Tissuespecific, hormonal, and developmental regulation of SCC-LacZ expression in transgenic mice leads to adrenocortical zone characterization. Endocrinology 140 5609-5618.

Hu MC, Hsu NC, Pai CI, Wang CK \& Chung B 2001 Functions of the upstream and proximal steroidogenic factor 1 (SF-1)-binding sites in the CYP11A1 promoter in basal transcription and hormonal response. Molecular Endocrinology 15 812-818.

Huang Y, Hu M, Hsu N, Wang CL \& Chung B 2001 Action of hormone responsive sequence in $2.3 \mathrm{~kb}$ promoter of CYP11A1. Molecular and Cellular Endocrinology 175 205-210.

Jefcoate CR, DiBartolomeis MJ, Williams CA \& McNamara BC 1987 ACTH regulation of cholesterol movement in isolated adrenal cells. Journal of Steroid Biochemistry 27 721-729.

John ME, John MC, Boggaram V, Simpson ER \& Waterman MR 1986 Transcriptional regulation of steroid hydroxylase genes by corticotropin. PNAS 83 4715-4719.

Kelly SN, McKenna TJ \& Young LS 2004 Modulation of steroidogenic enzymes by orphan nuclear transcriptional regulation may control diverse production of cortisol and androgens in the human adrenal. Journal of Endocrinology 181 355-365.

Kumar S \& Hedges SB 1998 A molecular timescale for vertebrate evolution. Nature 392 917-920.

Kuo MW, Postlethwait J, Lee WC, Lou SW, Chan WK \& Chung BC 2005 Gene duplication, gene loss and evolution of expression domains in the vertebrate nuclear receptor NR5A (Ftz-F1) family. Biochemical Journal 389 19-26.

Li LA, Chiang EF, Chen JC, Hsu NC, Chen YJ \& Chung BC 1999 Function of steroidogenic factor 1 domains in nuclear localization, transactivation, and interaction with transcription factor TFIIB and c-Jun. Molecular Endocrinology 13 1588-1598.

Li LA, Chang YC, Wang CJ, Tsai FY, Jong SB \& Chung BC 2004 Steroidogenic factor 1 differentially regulates basal and inducible steroidogenic gene expression and steroid synthesis in human adrenocortical H295R cells. Journal of Steroid Biochemistry and Molecular Biology 91 11-20.

Liu YW, Gao W, Teh HL, Tan JH \& Chan WK 2003 Prox1 is a novel coregulator of Fflb and is involved in the embryonic development of the zebra fish interrenal primordium. Molecular and Cellular Biology 23 7243-7255.

Luo X, Ikeda Y \& Parker KL 1994 A cell-specific nuclear receptor is essential for adrenal and gonadal development and sexual differentiation. Cell 77 481-490.

McGonnell IM \& Fowkes RC 2006 Fishing for gene function endocrine modelling in the zebrafish. Journal of Endocrinology 189 425-439.

Monte D, DeWitte F \& Hum DW 1998 Regulation of the human $\mathrm{P} 450 \mathrm{scc}$ gene by steroidogenic factor 1 is mediated by $\mathrm{CBP} / \mathrm{p} 300$. Journal of Biological Chemistry 273 4585-4591.

Morohashi K, Sogawa K, Omura T \& Fujii-Kuriyama Y 1987 Gene structure of human cytochrome P-450(SCC), cholesterol desmolase. Journal of Biochemistry 101 879-887. 
Oonk RB, Parker KL, Gibson JL \& Richards JS 1990 Rat cholesterol side-chain cleavage cytochrome P-450 (P-450scc) gene. Structure and regulation by cAMP in vitro. Journal of Biological Chemistry $\mathbf{2 6 5}$ 22392-22401.

Parker KL, Rice DA, Lala DS, Ikeda Y, Luo X, Wong M, Bakke M, Zhao L, Frigeri C, Hanley NA et al. 2002 Steroidogenic factor 1: an essential mediator of endocrine development. Recent Progress in Hormone Research 57 19-36.

Payne AH \& Hales DB 2004 Overview of steroidogenic enzymes in the pathway from cholesterol to active steroid hormones. Endocrine Reviews 25 947-970.

Pestell RG, Hammond VE \& Crawford RJ 1993 Molecular cloning and characterization of the cyclic AMP-responsive ovine CYP11A1 (cholesterol side-chain cleavage) gene promoter: DNase 1 protection of conserved consensus elements. Journal of Molecular Endocrinology 10 297-311.

Rice DA, Kirkman MS, Aitken LD, Mouw AR, Schimmer BP \& Parker KL 1990 Analysis of the promoter region of the gene encoding mouse cholesterol side-chain cleavage enzyme. Journal of Biological Chemistry 265 11713-11720.

Sadovsky Y, Crawford PA, Woodson KG, Polish JA, Clements MA, Tourtellotte LM, Simburger K \& Milbrandt J 1995 Mice deficient in the orphan receptor steroidogenic factor 1 lack adrenal glands and gonads but express $\mathrm{P} 450$ side-chain-cleavage enzyme in the placenta and have normal embryonic serum levels of corticosteroids. PNAS 92 10939-10943.

De Santa BP, Bonneaud N, Boizet B, Desclozeaux M, Moniot B, Sudbeck P, Scherer G, Poulat F \& Berta P 1998 Direct interaction of SRY-related protein SOX9 and steroidogenic factor 1 regulates transcription of the human anti-Mullerian hormone gene. Molecular and Cellular Biology 18 6653-6665.
Shen WH, Moore CC, Ikeda Y, Parker KL \& Ingraham HA 1994 Nuclear receptor steroidogenic factor 1 regulates the mullerian inhibiting substance gene: a link to the sex determination cascade. Cell 77 651-661.

Siebert PD, Chenchik A, Kellogg DE, Lukyanov KA \& Lukyanov SA 1995 An improved PCR method for walking in uncloned genomic DNA. Nucleic Acids Research 23 1087-1088.

de Souza BF, Lin L \& Achermann JC 2006 Steroidogenic factor-1 (SF-1) and its relevance to pediatric endocrinology. Pediatric Endocrinology Reviews 3 359-364.

Tremblay JJ \& Viger RS 1999 Transcription factor GATA-4 enhances Mullerian inhibiting substance gene transcription through a direct interaction with the nuclear receptor SF-1. Molecular Endocrinology 13 1388-1401.

Val P, Lefrancois-Martinez AM, Veyssiere G \& Martinez A 2003 SF-1 a key player in the development and differentiation of steroidogenic tissues. Nuclear Receptor 18.

Zhang P, Rodriguez H \& Mellon SH 1995 Transcriptional regulation of P450scc gene expression in neural and steroidogenic cells: implications for regulation of neurosteroidogenesis. Molecular Endocrinology 9 1571-1582.

Ben Zimra M, Koler M \& Orly J 2002 Transcription of cholesterol side-chain cleavage cytochrome $\mathrm{P} 450$ in the placenta: activating protein-2 assumes the role of steroidogenic factor- 1 by binding to an overlapping promoter element. Molecular Endocrinology 16 1864-1880.

Received in final form 26 April 2009

Accepted 26 May 2009

Made available online as an Accepted Preprint 28 May 2009 